\title{
Mucosal Neuroma Cues for Endocrine Emergency
}

\section{Treatment}

\author{
Gyu Gang Choi ${ }^{1}$, Hwan Jin Lee ${ }^{1}$, Hyo Jin Han ${ }^{1}$, Young Beom Jeong ${ }^{2}$, Heung Bum Lee ${ }^{1}$, Ji Hyun Park ${ }^{1}$ \\ Departments of ${ }^{1}$ Internal Medicine, ${ }^{2}$ Urology, Jeonbuk National University Medical School, Jeonju, Korea
}

A 34-year-old man presented with poor oral intake and abdominal pain with bilateral adrenal masses on abdominal computed tomography (CT). He had marfanoid features and mild intellectual disability. During the initial physical examination, lingual mucosal neuromas were identified, although they were obscured initially by a cracked and dry tongue, which are signs of dehydration (Fig. 1A); the neuromas with thickened lips were more clearly visible on a photograph taken 6 days after the bilateral adrenalectomy (Fig. 1B). Multiple endocrine neoplasia type 2B (MEN2B) was suspected. He had severe hypertension and hypokalemia of $2.4 \mathrm{mmol} / \mathrm{L}$, a transtubular potassium gradient of 9 , and high plasma renin activity, which was uncontrolled despite alpha-blocker and spironolactone treatment. The blood pressure was managed using an additional intravenous calcium channel blocker and beta-blocker infusion therapy, but it was very labile. The accompanying intestinal pseudo-obstruction worsened despite aggressive medical treatments, while the acalculous cholecystitis was managed via percutaneous transhepatic gallbladder drainage (PTGBD). Ectopic adrenocorticotropic hormone (ACTH) syndrome (EAS), a life-threatening endocrine emergency, was suspected based on a serum cortisol level $>75 \mu \mathrm{g} / \mathrm{dL}$, ACTH of $868 \mathrm{pg} / \mathrm{mL}$ (reference, 10 to 90 ), serum cortisol $>75 \mu \mathrm{g} / \mathrm{dL}$ after $1 \mathrm{mg}$ overnight dexamethasone sup-

Received: 17 September 2021, Revised: 25 October 2021,

Accepted: 4 November 2021

Corresponding author: Ji Hyun Park

Division of Endocrinology and Metabolism, Department of Internal Medicine, Jeonbuk National University Medical School, Research Institute of Clinical Medicine of Jeonbuk National University-Biomedical Research Institute of Jeonbuk National University Hospital, 20 Geonji-ro, Deokjin-gu, Jeonju 54907, Korea

Tel: +82-63-250-1780, Fax: +82-63-254-1609, E-mail: parkjh@jbnu.ac.kr pression, and the results of a corticotropin-releasing hormone stimulation test. A neck ultrasound showed four thyroid nodules, including a $1.8 \mathrm{~cm}$-sized nodule in the left thyroid gland (Fig. 2A), with cervical lymph nodes suspicious for metastasis. Cells aspirated from the nodule tested positive for calcitonin on immunocytochemical staining. Bilateral pheochromocytomas were suggested by abdominal CT (Fig. 2B) and 18F-fluorodeoxyglucose positron emission tomography with CT findings, as well as elevated levels of plasma metanephrine ( $>20 \mathrm{nmol} / \mathrm{L}$; reference, $\leq 0.5$ ), plasma normetanephrine ( $>45 \mathrm{nmol} / \mathrm{L}$; reference, $\leq 0.9)$, 24-hour urine vanillylmandelic acid (196 mg/day; reference, $\leq 8)$, and 24-hour urine metanephrine (49 mg/day; reference, $\leq 0.8$ ). Although either a pheochromocytoma or medullary thyroid carcinoma (MTC) are possible causes of EAS in MEN2B [1,2], bilateral adrenalectomy following medical control of intense hypercortisolism and preoperative alpha-blockade was considered first as a definitive treatment for bilateral pheochromocytoma; this can also serve as a rescue treatment in cases of EAS caused by MTC [3]. An etomidate infusion was started in the intensive care unit to inhibit steroidogenesis; 6 days later, bilateral adrenalectomy was performed successfully. After surgery, bowel distension was relieved, the blood pressure and biochemical parameters returned to normal, and the PTG-

Copyright $\odot 2021$ Korean Endocrine Society

This is an Open Access article distributed under the terms of the Creative Commons Attribution Non-Commercial License (https://creativecommons.org/ licenses/by-nc/4.0/) which permits unrestricted non-commercial use, distribution, and reproduction in any medium, provided the original work is properly cited. 

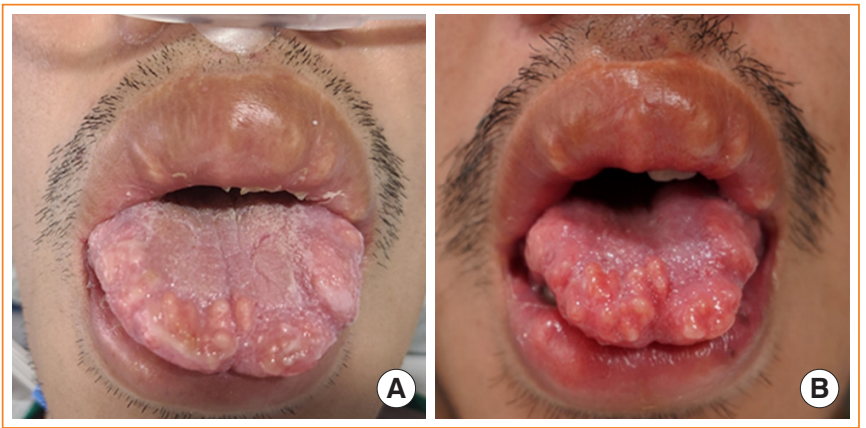

Fig. 1. (A) Mucosal neuromas on the tongue are found during the initial physical examination at the time of hospital admission, although a dehydrated, cracked, and dry tongue obscures this finding. A histopathological examination was not performed for these neuromas because they were benign tumors and had no specific symptoms. (B) A photograph, taken 6 days after the ectopic adrenocorticotropic hormone syndrome was resolved by bilateral adrenalectomy, shows clearly visible lingual mucosal neuromas with thickened lips, which are typical features of multiple endocrine neoplasia type 2B (MEN2B).

BD could be removed. After obtaining informed consent, RET proto-oncogene analysis confirmed an M918T mutation. Following discharge from the hospital, the patient has been receiving hydrocortisone replacement therapy, and his performance status is improving. Vandetanib was initiated to treat MTC with liver metastasis in which calcitonin levels exceed $8,000 \mathrm{pg} / \mathrm{mL}$ and carcinoembryonic antigen levels are elevated $(299 \mathrm{ng} / \mathrm{mL}$; reference, 0 to 5). The patient's calcitonin levels had decreased to $1,322 \mathrm{pg} / \mathrm{mL}$ at 1 month after the initiation of treatment. To successfully treat this rare, life-threatening endocrine emergency, a careful physical examination and timely multidisciplinary planning among several departments are essential.

This image of interest was approved by the Institutional Review Board (JNUH IRB approval no. 2021-09-031). Written informed consent was obtained from the patient and a family member to publish this image of interest.

\section{CONFLICTS OF INTEREST}

No potential conflict of interest relevant to this article was reported.

\section{ORCID}

Gyu Gang Choi https://orcid.org/0000-0003-2313-5322

Ji Hyun Park https://orcid.org/0000-0002-9430-8887
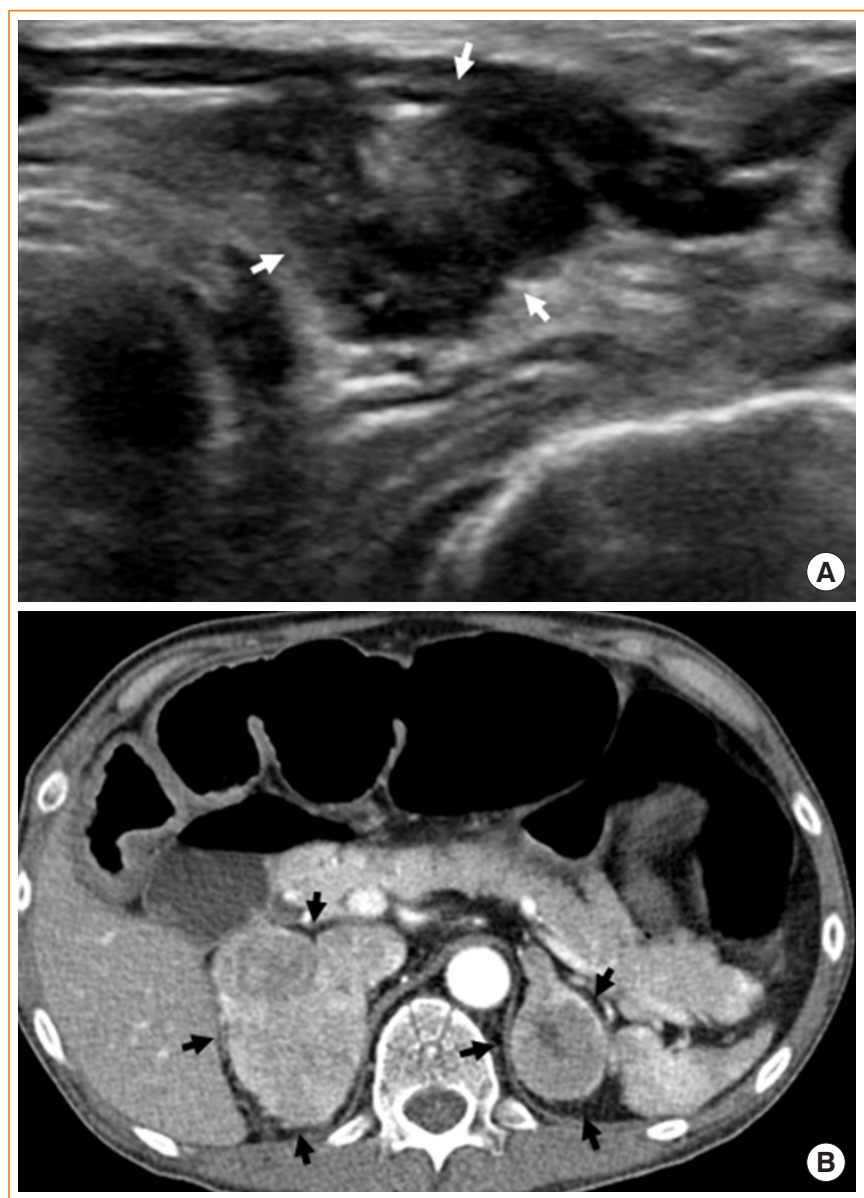

Fig. 2. (A) Transverse neck sonographic image showing a $1.8 \mathrm{~cm}$, irregular-shaped, hypoechoic nodule (white arrows) with speculated margins, heterogeneous echotexture of the solid portion, and microcalcifications located in the left lobe of the thyroid. (B) Axial contrast-enhanced computed tomography image showing heterogeneous enhancing masses in both adrenal glands (black arrows), including focal cystic components in the left adrenal mass, with diffuse dilatation of the transverse colon.

\section{REFERENCES}

1. Elliott PF, Berhane T, Ragnarsson O, Falhammar H. Ectopic ACTH- and/or CRH-producing pheochromocytomas. J Clin Endocrinol Metab 2021;106:598-608.

2. Choi HS, Kim MJ, Moon CH, Yoon JH, Ku HR, Kang GW, et al. Medullary thyroid carcinoma with ectopic adrenocorticotropic hormone syndrome. Endocrinol Metab (Seoul) 2014; 29:96-100.

3. Young J, Haissaguerre M, Viera-Pinto O, Chabre O, Baudin E, Tabarin A. Management of endocrine disease: Cushing's syndrome due to ectopic ACTH secretion: an expert operational opinion. Eur J Endocrinol 2020;182:R29-58. 